\title{
Apnoea characteristics across the night in severe obstructive sleep apnoea: influence of body posture
}

\author{
A. Oksenberg*, I. Khamaysi*, D.S. Silverberg ${ }^{\#}$
}

\begin{abstract}
Apnoea characteristics across the night in severe obstructive sleep apnoea: influence of body posture. A. Oksenberg, I. Khamaysi, D.S. Silverberg. (C) ERS Journals Ltd 2001. ABSTRACT: Several studies have reported changes in apnoea characteristics across the night in patients with obstructive sleep apnoea (OSA). However, the effect of body posture on these changes has not been evaluated. The purpose of this study was to assess how body posture influences sleep apnoea characteristics across the night in severe OSA.

Polysomnograms of 30 patients with severe OSA who had $\geqslant 30$ apnoeic episodes in the lateral position and 30 in the supine position during Stage 2 sleep, in the early, middle and late sleep periods were analysed. For each of the 30 events in each body position, the following variables were evaluated: apnoea duration; minimum saturation; change in saturation; duration of arousal; maximum snoring loudness and change in cardiac frequency.

Although at any time during the night, apnoeas in the supine posture were more severe than those in the lateral position, a time effect across the night was found only for apnoea duration, change in cardiac frequency and duration of arousal. Body position did not affect the time trend for these variables across the night. The best fit for the changes of these three variables across the night was a quadratic time trend.

It was concluded that in severe obstructive sleep apnoea during Stage 2 sleep, body position does not significantly affect the time trends of apnoea characteristics across the night. These data provide support for the participation of sleep-dependent mechanisms (homeostatic and circadian) in the determination of apnoea characteristics across the night.

Eur Respir J 2001; 18: 340-346.
\end{abstract}

*Sleep Disorders Unit, Loewenstein Hospital-Rehabilitation Centre, Raanana and ${ }^{\#}$ Dept of Nephrology, TelAviv Medical Centre, Tel-Aviv, Israel.

Correspondence: A. Oksenberg, Sleep Disorders Unit, Loewenstein HospitalRehabilitation Centre, POB 3 Raanana, Israel.

Fax: 97297709123

Keywords: Apnoea characteristics apnoea length

body posture

obstructive sleep apnoea

sleep position

Received: April 272000

Accepted after revision July 182001
In 1981, LAVIE et al. [1] described the lengthening of apnoeas across the night in patients with obstructive sleep apnoea (OSA). They studied eight patients who had $>200$ apnoeas per night and found a significant linear trend for increasing duration of apnoeas during Stage 2 sleep and for apnoeas in all sleep stages pooled together. No such findings were observed for rapid eye movement (REM) sleep or for the apnoea index (AI). The authors suggested a progressive increase in arousal threshold as the explanation for their findings. Recently, several studies have further investigated the lengthening of apnoeic events across the night in OSA patients in an attempt to better understand the mechanisms responsible.

Charbonneau et al. [2] found similar results and in addition, demonstrated that the end-apnoeic desaturation did not change across the night due to a decrease in the rate of desaturation as the night progressed. An increase in the time spent in apnoea was also observed, but worsening of AI across the night was only seen in patients with very severe OSA (AI>85). Unfortunately, in these two previous studies, body position was not taken in consideration. In order to better understand the mechanism of apnoea lengthening across the night, KImofF et al. [3] compared the maximal diaphragmatic tension-time index at end-apnoea during administration of air, oxygen $\left(\mathrm{O}_{2}\right)$, and carbon dioxide $\left(\mathrm{CO}_{2}\right)$ in seven untreated patients with severe OSA and found evidence supporting the hypothesis that apnoea termination is related more directly to the level of inspiratory effort than to the level of chemical stimulus. Subsequently, Montserrat et al. [4], measured transdiaphragmatic pressure and diaphragmatic tension-time index in seven patients with severe OSA during Stage 2 sleep, at the beginning and at the end of the night and in the supine position only. They observed an increase in apnoea duration with no significant change in end-apnoeic arterial oxygen saturation $\left(\mathrm{Sa}_{\mathrm{a}} \mathrm{O}_{2}\right)$, but they also showed that although the rate of increase in inspiratory effort was similar, the level of inspiratory effort at end-apnoea was substantially greater at the end of the night. Based on these and previous results, the authors proposed that a progressive decline in the arousal response (or an increase in arousal threshold) to neural stimuli generated during obstructive inspiratory efforts (probably mediated by mechanoreceptors) plays a major role in apnoea lengthening across the night.

The effect of body posture on apnoea lengthening has not been assessed. In some of the previous studies body posture was not even mentioned $[1,2,3]$ and in 
one [4], the evaluation was done in the supine posture only. It is possible that the degree of lengthening of apnoeic events differs in the supine posture compared to the lateral posture as the night goes on. The authors recently showed that supine apnoeic events are more severe than lateral apnoeic events [5]. It was also found that the supine posture increases the optimal nasal continuous positive airway pressure (nCPAP) by $>2.0 \mathrm{~cm} \mathrm{H} \mathrm{H}_{2} \mathrm{O}$ compared to the lateral posture [6]. This increase in optimal nCPAP for supine apnoeas implies an upper airway that is more difficult to reopen, therefore producing more severe apnoea. If supine apnoeas were indeed more detrimental than those occurring in the lateral posture, it could be expected that the degree of lengthening across the night of supine apnoeas would be more pronounced than for lateral apnoeas. It is possible that as the night goes on, it may become harder and harder to reopen the obstructed upper airway, thus causing a greater increase in the duration of these supine apnoeas in comparison to lateral apnoeas.

The aim of this study was to evaluate the effect of body posture on different aspects of apnoea across the night in patients with severe OSA.

\section{Patients and methods}

\section{Patients}

The polysomnograms of 30 patients with severe OSA (respiratory disturbance index $(\mathrm{RDI})=70.1 \pm 18.2$ mean $\pm \mathrm{SD})$, who had nonpositional sleep apnoea i.e. in whom the ratio of the supine RDI to the lateral RDI was $<2$ (supine RDI $=85.7 \pm 11.7$, lateral $\mathrm{RDI}=64.8 \pm$ 17.3) and $\geqslant 30$ apnoeas in the lateral position and 30 in the supine position during sleep Stage 2, were included in the study. Only one of the patients slept in the prone posture during polysomnography. This patient was included in the analysis but this prone period was eliminated from the analysis. In order to find 30 polysomnograms without major artefacts and with $\geqslant 30$ apnoeic episodes in both the lateral and supine positions during Stage 2 sleep, the polysomnographs of 92 consecutive OSA patients with nonpositional apnoea diagnosed in the authors unit had to be reviewed.

The 30 apnoeic events in each position included samples of 10 consecutive events in the initial, middle and final third of the total sleep period. Polysomnograms of patients with congestive heart failure or chronic obstructive pulmonary disease (COPD) were excluded from the study. The 30 patients (26 male and four female) had a mean \pm SD age of 57.6 \pm 11.7 and body mass index $(\mathrm{BMI})$ of $32.3 \pm 4.3 \mathrm{~kg} \cdot \mathrm{m}^{2}$.

Table 1 summarizes the demographic and sleep data of all 30 patients. On average, they spent more time sleeping in the lateral than in the supine posture. The sleep time in each third of the night was quite similar. The mean total sleep time (TST) for all subjects was $364.1 \pm 60.7 \mathrm{~min}$, (range, 211-470), the mean TST supine was $135.3 \pm 63.5 \mathrm{~min}$ (range, 51-320), and the mean TST lateral was $228.7 \pm 84.1 \mathrm{~min}$ (range, 28-392).
Table 1. - Summary of demographic, respiratory disturbance index and total sleep time data of the 30 patients with severe nonpositional obstructive sleep apnoea

\begin{tabular}{lc}
\hline & Mean \pm SD (Range) \\
\hline Age yrs & $57.6 \pm 11.7(26-74)$ \\
BMI & $32.3 \pm 4.3(24.5-47)$ \\
RDI-S & $85.7 \pm 11.7(45.8-157.3)$ \\
RDI-L & $64.8 \pm 17.3(40.3-105.9)$ \\
S:L & $1.3 \pm 0.2(1.1-1.7)$ \\
RDI-T & $71.3 \pm 16.7(44-112)$ \\
TST & $364.1 \pm 60.7(211-470)$ \\
TST-S & $135.3 \pm 63.5(51-320)$ \\
TST-L & $228.8 \pm 84.1(28-392)$ \\
ST-1/3 & $123.7 \pm 22.6(73-159)$ \\
ST-2/3 & $119.5 \pm 21.3(61-147)$ \\
ST-3/3 & $120.9 \pm 22.4(71-162)$ \\
\hline
\end{tabular}

BMI: body mass index; RDI-S: respiratory disturbance index in supine posture; RDI-L: respiratory disturbance index in lateral posture; S:L: ratio RDI-S/RDI-L; RDI-T: total respiratory disturbance index; TST: total sleep time; TST-S: supine total sleep time; TST-L: lateral total sleep time; ST-1/3: sleep time in the first third; ST-2/3: sleep time in the second third; ST-3/3: sleep time in the third third.

\section{Polysomnographic evaluation}

The patients arrived at the sleep unit around 20:00 $\mathrm{h}$ and the polysomnographic recordings usually began between 22:00 $\mathrm{h}$ and midnight. The recordings were carried out using Nihon Koden polygraphs (models 4321 and 4414; Nihon Koden, Tokyo, Japan) and included the following: electro-oculogram (EOG) (2-4 channels); electroencephalogram (EEG) (4-6 channels); electromyogram (EMG) of submental muscles (1-2 channels); electrocardiogram (ECG) (1 channel); EMG of the anterior tibialis muscle of both legs (2 channels); and airflow (with a nasal/oral thermistor). Chest and abdominal effort (2 channels) were recorded using inductive plethysmography (Respitrace-Ambulatory Monitoring Inc., Ardsley, New York or Resp-Ez breathing belts, Scientific Laboratory Products, Tel Aviv, Israel); $\mathrm{Sa}, \mathrm{O}_{2}$ was measured (1 channel) by pulse oximetry (Ohmeda 3700e, Boulder, CO, USA) using a finger probe; and snoring was recorded (1 channel) by a microphone located above the patient's head at a distance of 1 metre and connected to a sound level metre (SLM) (model 2700; Quest Electronics, Oconomowoc, WI, USA). The output from the SLM was also recorded in parallel on a calibrated $(40-80 \mathrm{~dB})$ chart recorder at a paper speed of $10 \mathrm{~cm} \cdot \mathrm{h}^{-1}$. The recordings were carried out at a paper speed of $10 \mathrm{~mm} \cdot \mathrm{s}^{-1}$ and sleep was scored according to the standard criteria of ReCHTSCHAFFEN and KALES [7]. The polysomnographic technician who observed the patient's behaviour on a closed circuit 21 inch television monitor, marked the changes in body position in two places simultaneously, on the polygraph and on the chart recorder which registered the output of the pulse oximeter data. The technician was responsible for monitoring one or two sleeping patients at a time. The two monitors were placed side-by-side to allow easy 
visualization of all patients' body movements. Since the authors unit is especially interested in the effect of body position on sleep-related breathing disturbances, the technicians were encouraged to pay special attention to this. Obstructive apnoea was defined as an episode of complete cessation of breathing of $\geqslant 10 \mathrm{~s}$ with continuing inspiratory effort. Arousals were scored according to accepted criteria [8].

\section{Selection of apnoeic events}

The procedure for selection of apnoeas was not random, but they were selected in a consecutive manner throughout the polysomnogram. For each one-third of the record, a continuous period of Stage 2 sleep was first identified (without major interruptions with Stage 1, Stage 0 or movement time) with apnoeic episodes. This Stage 2 sleep period occurred before or after an REM period. The analysis of apnoeas was restricted only to epochs of Stage 2 sleep. The body posture was then identified and 10 consecutive apnoeas (without artifacts) were selected from the beginning of that Stage 2 sleep period. A period of Stage 2 sleep in the other posture was then identified and 10 consecutive apnoeas were selected from the beginning of that period. The same selection procedure was used for the second and last third of the polysomnographic record.

\section{Analysis of apnoeic events}

Six variables related to each apnoea, were evaluated in the polysomnograms of the 30 OSA patients. For each patient, 30 events in the supine posture and 30 in the lateral posture occurring in sleep Stage 2 throughout the night, were analysed visually by one of the authors for the following parameters: apnoea duration, minimum saturation, change in $\mathrm{O}_{2}$ saturation (the difference between minimum and maximum $\mathrm{Sa}_{\mathrm{a}} \mathrm{O}_{2}$ ), duration of arousal, maximum snoring loudness, and change in cardiac frequency (the difference between the heart rate $3 \mathrm{~s}$ before and $3 \mathrm{~s}$ after the end of the apnoea).

\section{Statistical analysis}

To investigate whether a time trend across the night for the several variables of apnoea events existed, analysis of variance (ANOVA) for repeated measures was performed. The within subject factors were position and time. This analysis enabled the examination of the overall differences between the two body positions and the presence of a linear and/or a quadratic time trend and the interaction between position and time for each variable. The time trend for the different variables was studied by applying polynomial equations to the repeated measures. Trend analysis is a common procedure for withinsubject (serial) designs, and is usually part of an ANOVA with repeated measures. Its purpose is to determine the type of time trend (linear, quadratic, cubic, etc.) that gives the best fit for the data. The highest polynomial degree that can be fitted to the data is determined by the number of levels of the within-subject factor: it equals the number of levels minus 1 . Therefore, data generated from a study with a 3-level factor can be fitted by a quadratic polynomial at most [9].

Since there were only three time points, only the linear and quadratic polynomials could be examined. A nonsignificant result of the F-test indicates that the corresponding polynomial is a good fit for the data. The values are expressed as mean \pm SD. A p-value $<0.05$ was considered to be significant.

\section{Results}

Table 2 summarizes the values of each third of the night for all apnoea parameters in the supine and lateral posture. With repeated measures the ANOVA first of all revealed that for all apnoea variables assessed, an overall significant difference was found between the two body positions (table 3 ). The supine duration was longer than the lateral duration ( $p=0.0001$ ); the supine minimum saturation was less than that in the lateral position $(\mathrm{p}=0.0001)$; the supine change in saturation was greater than that in the lateral position $(p=0.0001)$; the supine change in cardiac frequency was greater than that in the lateral $(\mathrm{p}=0.0001)$; the maximum snoring loudness was greater in the supine than the lateral position $(\mathrm{p}=0.04)$; and the duration of arousals was much longer in the supine than in the lateral position $(\mathrm{p}=0.0001)$.

An overall time effect was found for only three apnoea variables (table 3): apnoea duration $(\mathrm{p}=$ $0.0001)$; change in cardiac frequency $(\mathrm{p}=0.03)$; and duration of arousal $(\mathrm{p}=0.053)$. Each of these variables

Table 2. - Apnoea variables in supine and lateral positions according to thirds of the night

\begin{tabular}{lccccccc}
\hline Position & $\begin{array}{c}\text { Thirds of } \\
\text { night }\end{array}$ & $\begin{array}{c}\text { Apnoea } \\
\text { duration }\end{array}$ & $\begin{array}{c}\text { Minimum } \\
\text { saturation }\end{array}$ & $\begin{array}{c}\text { Change in } \\
\text { saturation }\end{array}$ & $\begin{array}{c}\text { Duration of } \\
\text { arousal }\end{array}$ & $\begin{array}{c}\text { Maximum snoring } \\
\text { loudness }\end{array}$ & $\begin{array}{c}\text { Change in cardiac } \\
\text { frequency }\end{array}$ \\
\hline Supine & $1 \mathrm{st}$ & $24.7 \pm 6.1$ & $82.4 \pm 7.3$ & $12.0 \pm 5.2$ & $7.8 \pm 2.3$ & $60.0 \pm 13.5$ & $87 \pm 3.8$ \\
& 2nd & $26.3 \pm 7.9$ & $82.0 \pm 6.9$ & $12.6 \pm 5.5$ & $8.9 \pm 2.9$ & $63.8 \pm 7.9$ & $9.1 \pm 4.0$ \\
& 3rd & $27.9 \pm 7.1$ & $81.5 \pm 7.2$ & $13.4 \pm 5.9$ & $8.9 \pm 2.1$ & $63.6 \pm 7.2$ & $9.4 \pm 4.1$ \\
Lateral & $1 \mathrm{st}$ & $21.4 \pm 4.8$ & $86.4 \pm 5.5$ & $8.1 \pm 4.1$ & $4.7 \pm 1.3$ & $59.9 \pm 7.5$ & $7.5 \pm 3.3$ \\
& 2nd & $23.4 \pm 5.1$ & $86.1 \pm 5.5$ & $8.3 \pm 3.7$ & $4.9 \pm 1.6$ & $61.8 \pm 7.2$ & $7.0 \pm 38$ \\
\hline
\end{tabular}

Data are presented as mean $\pm \mathrm{SD}$. 
Table 3. - Effect of position, time and position-time interaction on apnoea variables

\begin{tabular}{|c|c|c|c|c|c|}
\hline & Position & Time & Position-time interaction & Time 1 & Time 2 \\
\hline \multicolumn{6}{|c|}{ Apnoea duration } \\
\hline F-test & 41.23 & 15.71 & 1.19 & 12.82 & 5.33 \\
\hline p-value & 0.0001 & 0.0001 & 0.326 & 0.0012 & 0.028 \\
\hline \multicolumn{6}{|c|}{ Minimum saturation } \\
\hline F-test & 42.86 & 0.875 & 0.406 & 1.55 & 0.43 \\
\hline $\mathrm{p}$-value & 0.0001 & 0.43 & 0.67 & 0.22 & 0.52 \\
\hline \multicolumn{6}{|c|}{ Change in saturation } \\
\hline F-test & 46.92 & 1.90 & 1.16 & 2.08 & 2.2 \\
\hline $\mathrm{P}$-value & 0.0001 & 0.168 & 0.33 & 0.16 & 0.15 \\
\hline \multicolumn{6}{|c|}{ Change in cardiac frequency } \\
\hline F-test & 58.90 & 3.95 & 0.133 & 4.25 & 0.72 \\
\hline p-value & 0.0001 & 0.03 & 0.88 & 0.048 & 0.40 \\
\hline \multicolumn{6}{|c|}{ Maximum snoring loudness } \\
\hline F-test & 4.44 & 2.53 & 0.49 & 4.96 & 0.00 \\
\hline p-value & 0.04 & 0.098 & 0.62 & 0.03 & 0.97 \\
\hline \multicolumn{6}{|c|}{ Duration of arousal } \\
\hline F-test & 148.5 & 3.27 & 1.63 & 5.76 & 0.11 \\
\hline p-value & 0.0001 & 0.053 & 0.21 & 0.023 & 0.74 \\
\hline
\end{tabular}

Time 1: difference between the second and the first third of the night; Time 2: difference between the third and the second third of the night.

showed an increase as the night progressed. No significant interaction between time and position was found for any of the apnoea variables (table 3), implying that the time trend for each apnoea variable was not significantly affected by the body position. Thus, in spite of the impression that the time trend for supine and lateral apnoeas appears to be different (mainly for apnoea duration), these differences did not reach, statistical significance (fig. 1).

In addition, contrast analysis for the successive differences between the three time intervals were assessed and compared for the two body positions (table 3). This analysis shared an overall significant difference for apnoea duration, between the second and the first third $(\mathrm{p}=0.001)$ and between the final and second third $(\mathrm{p}=0.03)$; for change in cardiac frequency there was a difference only between the second and the first third $(p=0.05)$ and also between the second and the first third $(\mathrm{p}=0.02)$ for duration of arousal. In this analysis, no significant interaction between time and position was found for any of these three apnoea variables, implying that these time differences were consistent for the two body positions.

The correlation (Pearson correlation) between TST supine or lateral, RDI supine or lateral and the degree of apnoea lengthening across the night was also investigated. No significant correlation was found between supine or lateral sleep time and the degree of apnoea lengthening across the night in either position ( $\mathrm{r}=0.035, \mathrm{p}=0.85 ; \mathrm{r}=0.08, \mathrm{p}=0.66$ respectively $)$.

The polynomial contrast best fit for the three apnoea variables that showed a significant time trend across the night was also studied. The best fit for changes in time of the overall apnoea duration, change in cardiac frequency and duration of arousal data across the night was a quadratic function (table 4). Also, in this analysis no significant interaction between time and position was found for any of these three variables.

\section{Discussion}

The results of this study showed that during Stage 2 sleep in patients with severe OSA, the characteristics of apnoea events changed across the night and that they were in part related to the body posture. Apnoeas occurring in the supine posture are more severe than those occurring in the lateral position not only for the whole night [5], but also when this comparison is performed in each third of the night. However, body position does not significantly affect the time trend changes of the apnoea variables across the night. It should, however, be stressed that these results apply only to patients with severe $(\mathrm{RDI} \geqslant 40)$ nonpositional OSA.

Various authors have previously demonstrated a lengthening in apnoea duration across the night [1-4]. In the present study, these previous results were confirmed but they also showed that this increase in apnoea duration across the night is true for apnoea events in sleep Stage 2 occurring in the supine posture, as well as for events in the lateral posture.

Since supine apnoeic events are more severe than those in the lateral position [5, 6], it was speculated that the increase in severity of these supine apnoeas would have a more detrimental effect on the mechanisms responsible for the arousal, which terminates the apnoea and, consequently, would show a greater increase in duration across the night than lateral apnoeas. The results of the present study do not support this speculation. The fact that both supine and lateral apnoeas increase in duration overnight in a similar fashion, suggests that the harmful effect of supine apnoeas is certainly not the most important mechanism responsible for the lengthening of apnoeas across the night.

However, the results should be interpreted with caution. It is possible that because of small numbers a real increase in apnoea duration across the night in the 
a)

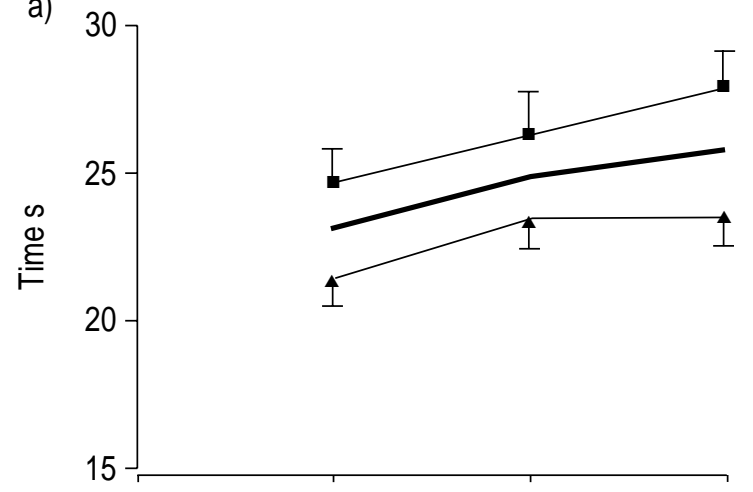

b)

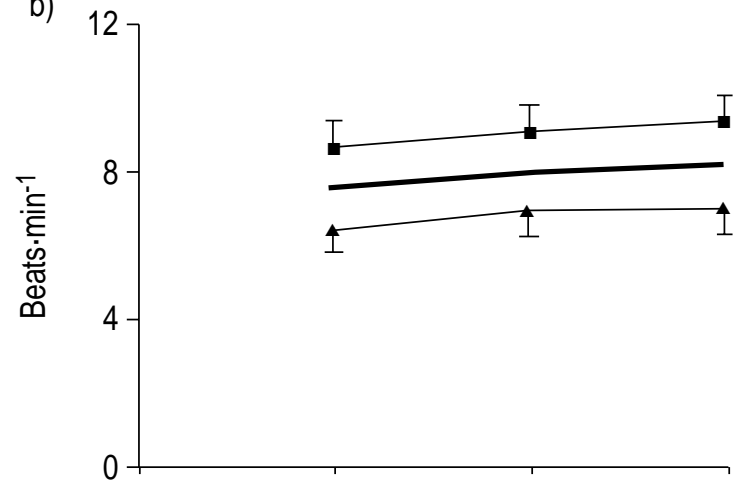

C)

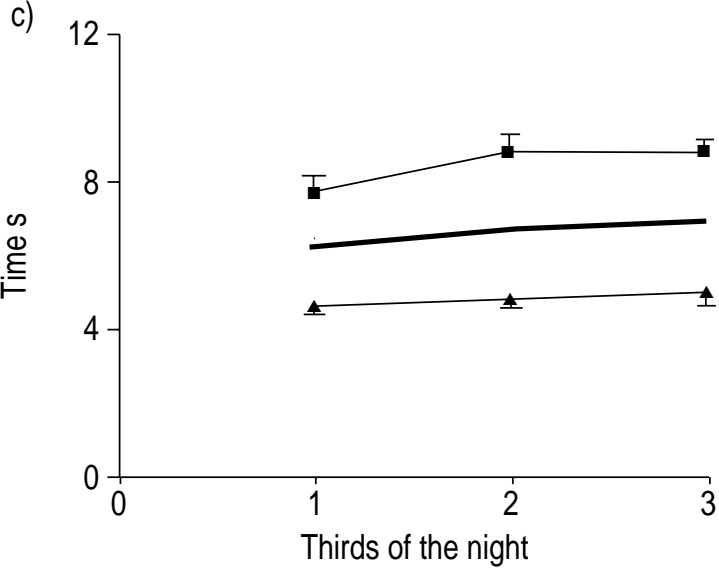

Fig. 1.-Time-trend across the night of three apnoea variables. a) Apnoea duration, b) change in cardiac frequency (the difference between the cardiac frequency value $3 \mathrm{~s}$ before and $3 \mathrm{~s}$ after the end of the apnoea event) and c) duration of arousal at the end of the apnoea. Significant increases with time across the night were found for each of these variables. Although at any time during the night, the apnoeas in the supine posture were more severe, no significant differences were observed for the time trends of these variables between supine and lateral apnoeas. Values are mean \pm SE. The bold lines denote the overall trends across the night without differentiating between body posture. $\boldsymbol{\square}$ : supine; $\boldsymbol{\Delta}$ : lateral.

supine compared to the lateral position was missed. In fact, there was a trend for increasing duration of apnoea in the supine position. However, the difference was small and even with 30 patients, the study had an $80 \%$ power of detecting a difference of $2.5 \mathrm{~s}$ in the change from the first to the third period (third) between supine and lateral apnoeas. Furthermore, there was no correlation between the increased duration of supine apnoea across the night and the time spent sleeping in the supine position. Therefore, it is unlikely that sleep position results in any clinically important differences in the duration of apnoea over the sleep period.

The data showing a quadratic trend for several apnoea parameters across the night should be interpreted with caution since there were only three points (the three thirds of the night) for this analysis and this allowed the authors to estimate only a linear or a quadratic type of change.

The mechanism responsible for the lengthening of apnoea across the night has not yet been elucidated. LAVIE et al. [1] suggested that the significant fragmentation which characterized OSA could produce a progressive delay in the arousal response that terminates apnoea and, therefore, could be the mechanism responsible for this increase in apnoea duration across the night. CHARBONNEAU et al. [2] suggested a decrease in respiratory muscle effort across the night as one of the mechanisms that could explain the increase in apnoea duration as the night progressed. The same group [3] showed that apnoea termination appeared to be best related to the level of inspiratory effort at end-apnoea, rather than to the level of chemical stimulus. In a further study [4], these authors obtained evidence that the level of inspiratory effort at end-apnoea increased across the night but the level of end-apnoea $\mathrm{Sa}_{\mathrm{a}} \mathrm{O}_{2}$ remained the same. They proposed that a progressive decline in the arousal response to neural stimuli generated during obstructed inspiratory efforts had a major role in apnoea lengthening across the night.

CALA et al. [10] showed that apnoea lengthening across the night could also be produced by local anaesthesia-induced blockade of upper airway (UA) mechanoreceptors at the beginning of the night. These findings support the authors' suggestion of progressive attenuation of function of UA mechanoreceptors across the night. They suggested that this may be a consequence of repeated trauma of pharyngeal tissue due to UA vibration and closure, and that it has an important role in the mechanism of apnoea lengthening.

The mechanisms responsible for the lengthening of apnoea across the night in OSA, therefore, appear to be complex. Sforza et al. [11] showed that the prolongation of apnoea was accompanied by an increase in peak inspiratory effort irrespective of changes in hypoxaemia, respiratory drive and pulmonary resistance, supporting the hypothesis that changes in arousal threshold are more directly implicated in the lengthening of apnoea than changes in UA collapsibility or chemosensitivity. The same group [12] reviewed 37 polysomnograms of OSA patients and analysed apnoea duration hour-by-hour and the maximal respiratory effort at the end of apnoea as judged by oesophageal pressure ( $\left.P_{\text {oes,max }}\right)$, an index of the arousal threshold. They found that both apnoea duration and $P$ oes,max increased during the first $3 \mathrm{~h}$ of sleep, showed a relatively stable level in the next $3 \mathrm{~h}$ and then a trend to decrease in those patients who slept for $8 \mathrm{~h}$. Since this trend was not associated with changes in arousability (arousal 
Table 4.-Polynomial best fit for the apnoea vairiables which showed a time trend increase across the night

\begin{tabular}{|c|c|c|c|c|c|c|}
\hline & \multicolumn{2}{|c|}{ Apnoea duration } & \multicolumn{2}{|c|}{ Change in cardiac frequency } & \multicolumn{2}{|c|}{ Duration of arousal } \\
\hline & F-test & $\mathrm{p}$-value & F-test & p-value & F-test & p-value \\
\hline Linear Fit & 32.2 & 0.0001 & 7.98 & 0.008 & 5.49 & 0.02 \\
\hline Quadratic Fit & 1.54 & 0.22 & 0.66 & 0.42 & 1.93 & 0.17 \\
\hline Position-time interaction & \multirow{2}{*}{\multicolumn{2}{|c|}{$\begin{array}{c}\text { NS } \\
\text { Quadratic }\end{array}$}} & \multirow{2}{*}{\multicolumn{2}{|c|}{ Quadratic }} & \multirow{2}{*}{\multicolumn{2}{|c|}{ Quadratic }} \\
\hline Best fit & & & & & & \\
\hline
\end{tabular}

A nonsignificant result of the F-test indicates that the corresponding polynomial is a good fit for the data. NS: nonsignificant.

density remained fairly constant across the night), they suggest that sleep-dependent mechanisms (homeostatic and circadian processes) may be more responsible for the nocturnal profile of arousal threshold to respiratory stimuli than the degree of sleep fragmentation. BERRY et al. [13] recently showed that the trend across the night of $P_{\text {oes,max }}$ closely parallels REM/non-REM sleeping cycling through the night, in parallel with the delta power, reaching the peak at or after the midcycle. These data provide further evidence for the important contribution of sleep-dependent mechanisms other than sleep fragmentation in the arousal responsiveness to respiratory stimuli across the night.

The findings that supine and lateral apnoea lengthen in a similar fashion from the first to the second third of the night and afterwards tend to reach a plateau, support the importance of sleep-related mechanisms in the control of arousal threshold to respiratory stimuli as suggested by SFORZA et al. [12]. In spite of the fact that supine apnoeas are more severe (are longer, generate more brady/tachycardia changes, cause longer arousal and more awakenings, produce more pronounced desaturations and louder end-apnoea snoring) than lateral apnoeas [5], both showed a similar trend of increased duration across the night (mainly during the first third of the night) probably due to the dominant control of arousability by the homeostatic (expressed by slow-wave activity) sleep-related mechanism. The participation of intrinsic sleep-related mechanisms in the control of arousal level to a respiratory stimulus does not preclude the active participation of other mechanisms such as the degree of sleep fragmentation, the changes in respiratory effort or the progressive attenuation of mechanoreceptor function. However, the present data and the data of other investigators $[12,13]$ suggest that these other mechanisms may only modulate the main sleepdependent mechanism in the control of arousability of respiratory stimuli across the night.

In summary, it has been shown that body position does not significantly affect the time trend of apnoea characteristics across the night during Stage 2 sleep in patients with severe nonpositional obstructive sleep apnoea. For those apnoea variables that are influenced by time across the night (apnoea duration, change in cardiac frequency and duration of arousals), no significantly different trends occurred for supine and lateral apnoeas. The evolution of each of these three apnoea variables during the night showed an overall quadratic time trend as the best fit. The results support the possible involvement of sleep-dependent mechanisms (homeostatic and circadian) in the determination of apnoea characteristics across the night.

\begin{abstract}
Acknowledgements. The authors would like to thank E. Shabtai for contribution in the statistical analysis and S. Melamed for valuable comments in the preparation of the manuscript.
\end{abstract}

\section{References}

1. Lavie P, Halperin E, Zomer J, Alroy G. Across-night lengthening of sleep apnoeic episodes. Sleep 1981; 4: 279-282.

2. Charbonneau M, Marin JM, Olha A, Kimoff J, Levy RD, Cosio MG. Changes in obstructive sleep apnoea characteristics through the night. Chest 1994; 106: 1695-1701.

3. Kimoff RJ, Cheong TH, Olha A. Mechanism of apnoea termination in obstructive sleep apnoea: role of chemoreceptor and mechanoreceptor stimuli. Am J Resp Crit Care Med 1994; 149: 707-714.

4. Montserrat JM, Kosmas EN, Cosio MG, Kimoff RJ. Mechanism of apnoea lengthening across the night in obstructive sleep apnoea. Am J Resp Crit Care Med 1996; 154: 988-993.

5. Oksenberg A, Khamaysi I, Silverberg DS, Tarasuik A. The association of body position with the severity of apnoea events in severe non-positional obstructive sleep apnoea (OSA) patients. Chest 2000; 118: 10181024.

6. Oksenberg A, Silverberg DS, Arons E, Radian H. The sleep supine position has a major effect on optimal nasal continuous positive airway pressure: Relationship with rapid eye movements and non-rapid eye movements sleep, body mass index, respiratory disturbance index and age. Chest 1999; 116: 10001006.

7. Rechtschaffen A, Kales A. A manual of standardized terminology techniques and scoring system for sleep stages of human subjects. LA, Brain Information Service/Brain Research Institute, University of California at Los Angeles, 1968.

8. American Sleep Disorders Association. EEG arousals: Scoring rules and examples. Sleep 1992; 15: 173-184.

9. Winer BJ, Brown DR, Michels KM. Statistical Principles in Experimental Design. New York, McGraw Hill, 1991; p. 199.

10. Cala SJ, Sliwinsky P, Cosio MG, Kimoff RJ. Effect of topical upper airway anaesthesia on apnoea duration 
through the night in obstructive sleep apnoea. $J$ Appl Physiol 1996; 81: 2618-2626.

11. Sforza E, Krieger J, Petiau C. Nocturnal evolution of respiratory effort in obstructive sleep apnoea syndrome: influence on arousal threshold. Eur Respir $J$ 1998; 12: 1257-1263.

12. Sforza E, Krieger J, Petiau C. Arousal threshold to respiratory stimuli in OSA patients: Evidence of a sleep-dependent temporal rhythm. Sleep 1999; 22: 6975.

13. Berry RB, Asyali MA, McNellis MI, Khoo MCK. Within-night variation in respiratory effort preceding apnoea termination and EEG delta power in sleep apnoea. J Appl Physiol 1998; 85: 1434-1441. 